\title{
CRIMALDDI: platform technologies and novel anti-malarial drug targets
}

\author{
Henri Vial ${ }^{1}$, Donatella Taramelli ${ }^{2}$, Ian C Boulton ${ }^{3}$, Steve A Ward ${ }^{4 *}$, Christian Doerig ${ }^{5}$ and Kelly Chibale ${ }^{6}$
}

\begin{abstract}
The Coordination, Rationalization, and Integration of antiMALarial drug Discovery \& Development Initiatives (CRIMALDDI) Consortium, funded by the EU Framework Seven Programme, has attempted, through a series of interactive and facilitated workshops, to develop priorities for research to expedite the discovery of new antimalarials. This paper outlines the recommendations for the development of enabling technologies and the identification of novel targets.

Screening systems must be robust, validated, reproducible, and represent human malaria. They also need to be cost-effective. While such systems exist to screen for activity against blood stage Plasmodium falciparum, they are lacking for other Plasmodium spp. and other stages of the parasite's life cycle. Priority needs to be given to developing high-throughput screens that can identify activity against the liver and sexual stages. This in turn requires other enabling technologies to be developed to allow the study of these stages and to allow for the culture of liver cells and the parasite at all stages of its life cycle.

As these enabling technologies become available, they will allow novel drug targets to be studied. Currently antimalarials are mostly targeting the asexual blood stage of the parasite's life cycle. There are many other attractive targets that need to be investigated. The liver stages and the sexual stages will become more important as malaria control moves towards malaria elimination. Sexual development is a process offering multiple targets, even though the mechanisms of differentiation are still not fully understood. However, designing a drug whose effect is not curative but would be used in asymptomatic patients is difficult given current safety thresholds. Compounds active against the liver schizont would have a prophylactic effect and Plasmodium vivax elimination requires effectors against the dormant liver hypnozoites. It may be that drugs to be used in elimination campaigns will also need to have utility in the control phase. Compounds with activity against blood stages need to be screened for activity against other stages.

Natural products should also be a valuable source of new compounds. They often occupy non-Lipinski chemical space and so may reveal valuable new chemotypes.
\end{abstract}

Keywords: CRIMALDDI, Malaria, Drug discovery, Research agenda, Novel targets, Enabling technologies, Prioritization

\section{Background}

There has been significant progress in recent years towards the global goal of malaria elimination. However, the recent reports of artemisinin resistance in Southeast Asia have emphasized the continuing need for a wide range of anti-malarials with novel chemical scaffolds and modes of action. This is needed to adequately address

\footnotetext{
* Correspondence: saward@liverpool.ac.uk

${ }^{4}$ Liverpool School of Tropical Medicine, Pembroke Place, Liverpool L3 5QA, UK

Full list of author information is available at the end of the article
}

the challenges to current drugs and extend the armoury to those aspects that are not well served by current drugs (especially against Plasmodium vivax malaria and transmission of the disease). The ultimate goal of elimination demands a range of new drugs must be developed quickly to meet very different challenges compared to control.

A major challenge for successful anti-malarial drug discovery is to quickly identify druggable targets and leads. This requires improved methods to study the various stages of the parasite life cycle (e, g. liver and blood stages, sexual forms, and midgut sporozoites) so as to

\section{Biomed Central}


find possible new drug targets. Some species and/or life cycle stages are difficult to maintain or produce ex vivo. Research on human malaria is hampered by technological difficulties that limit the study of parasite biology on all malaria stages and disease pathogenesis. Specialized laboratories or infrastructure 'platforms' are thus required to provide access to particular life cycle stages, and these methods need to be robust enough to be used in a wide range of laboratories.

As the objective of malaria treatment moves from control to elimination [1,2], the types of drugs required are changing. Drugs that block the infectivity of the mature sexual form of the gametocyte [3] and or the dormant hepatic form (hypnozoites) of P. vivax [4] will be particularly important. There will be questions about the balance between further exploiting existing drug targets and investing in identifying potential new ones, given the high costs/risks associated with new drug discovery and development. Other unanswered questions include the best way to identify new drug targets, whether targets that are common to several life-cycle stages are better than stage-specific targets, and how best to evaluate and prioritize new candidate compounds.

Most currently available anti-malarials target the asexual blood stages of the parasite's life cycle, the only stages for which there are robust, reproducible, and high-throughput in vitro screening methods. However, control and elimination will require new classes of anti-malarial drugs active against all stages of all five species of human malaria. Especially for elimination, new drugs are needed to eliminate all malaria parasites, including the $P$. vivax hypnozoites to prevent relapse, and to interfere with malaria transmission by eliminating gametocytes. Techniques to rapidly screen large libraries of compounds against targets at all the stages of the parasite life cycle of all relevant Plasmodium species are not currently available.

As described elsewhere, the Coordination, Rationalization, and Integration of antiMALarial drug Discovery \& Development Initiatives (CRIMALDDI) Consortium was established to develop a prioritized set of recommendations to expedite anti-malarial drug discovery [5]. This paper summarizes the findings of two of the Consortium's workshops organized to develop a set of consensus recommendations regarding enabling technologies and identifying novel targets. Full reports on the workshops can be found on the CRIMALDDI website [6] and in the related paper accompanying this one [7].

\section{Developing enabling technologies}

\section{General requirements for screening methods}

Screening to identify potential drug development compounds active against the various stages of the Plasmodium parasite life cycle can be viewed as a two-stage process (Figure 1). Primary screens must be simple enough to be used easily in high throughput screening systems (HTS), whereas secondary screens can be more complex and have only medium throughput capacity. For blood stage Plasmodium falciparum positive HTS hits, the current need is to filter $>20,000$ compounds from HTS whose structures are in the public domain down to $<200$ compounds to be considered for further development [8].

Primary screening methods must be robust, validated, reproducible, and represent human malaria (Table 1). Whole cell assays are a good way of identifying chemotypes with cellular permeability properties compatible with accessing their targets inside the parasite in its various stages. Screening systems must accommodate inherent strategies to minimise false negative results.

Methods primarily designed to determine the mode of action of a chemotype can be more complex and are implemented in later stages of the drug development process.

Additional requirements of primary screening should include:

- Cost-effectiveness;

- Biologically appropriate, i.e. the compounds actually kill the parasite (cidal rather than cytostatic);

- Small amount of compound needed for the screen;

- Very early exclusion of compounds cytotoxic against the human host;

- The screening process must include reliable models for all stages of the parasite's life cycle;

- Candidate compounds can either be schizonticidal plus active against another stage, or only active against another stage. Purely schizonticidal drugs are probably going to be of less interest as malaria control and elimination progresses. Primary screening must take this into account. However the rapid spread of resistance to current first-line drugs will require the deployment of curative agents that will probably be schizonticidal.

Secondary screening of positive hits from a primary screens can be more biologically informed and related to the desired activity of the target profile being pursued. Physiochemical and drug-like properties (including toxicological profile and pharmacokinetic parameters) must be considered since they should allow translation to in vivo models. Secondary screening must also look for activity against drug-resistant strains of Plasmodium and the capacity to withstand resistance. At present, routine screening is performed against parasite strains that obviously do not represent the strains circulating in the population. Screens need to have early access to isolates from patients, especially those showing resistance to important agents (such as artemisinin derivatives), and the 


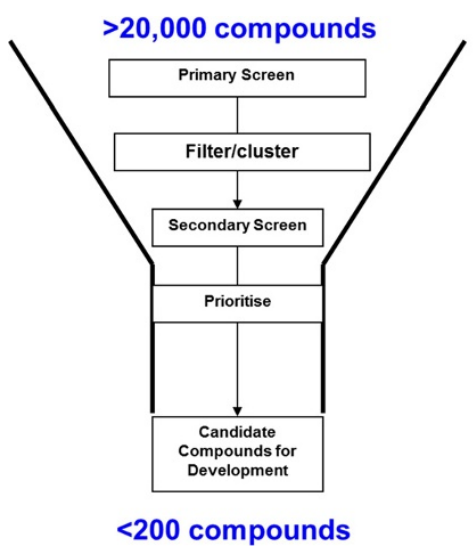

Primary Screens:

- Cost-effective

- Parasite is killed

- Small compound requirements

- Exclude cytotoxic compounds

- Identify activity vs. all stages of parasite lifecycle

- Define phenotype

Secondary Screens:

- Lower throughput?

- More biologically relevant to desired mode of action

- Translate to in vivo models

- Identify activity vs. resistant strains

- Define phenotype

Figure 1 Two-stage screening process for activity against the various stages of the Plasmodium parasite life cycle.

culture-adapted P. falciparum and P. vivax isolates used need to be updated regularly and promptly to reflect the changing parasite population in the field.

All screening methods need to be validated and reproducible between centres to ensure comparability of results [9].

\section{Screening methods against blood stage parasites}

The rapid identification and progression to drug development of compounds active against acute blood stage $P$. falciparum infections was made possible by the availability of an adequate range of in vitro screening assays and animal models. In contrast, there are significant gaps in the platform technologies necessary for investigating other species of Plasmodium, particularly P. vivax (Table 2).

\section{Plasmodium falciparum}

Along with the classic radioactive HTS screen for P. falciparum using ${ }^{3} \mathrm{H}$-hypoxanthine, colorimetric (pLDH, HRPII) [10-13] or fluorescent (SybrGreen) [14] assays have been widely used. Recently screens using transgenic parasites (GFP, Luciferase reporter strains) have been adapted to HTS [15-17]. At present, there is little need

\begin{tabular}{l}
$\begin{array}{l}\text { Table } 1 \text { Essential characteristics of screening assays for } \\
\text { novel compounds effective against the different stages } \\
\text { of malaria parasite life cycle }\end{array}$ \\
$\begin{array}{ll}\text { Robust } & \begin{array}{l}\text { Ensures that screens are easy to perform, } \\
\text { low cost, suitable for screening a large variety } \\
\text { of chemotypes or natural products. }\end{array} \\
\text { Ensures that the results reflect well the activity } \\
\text { of the compound against the targeted parasite } \\
\text { stage. }\end{array}$ \\
$\begin{array}{ll}\text { Ensures that the results obtained from different } \\
\text { Reproducible }\end{array}$ \\
$\begin{array}{l}\text { laboratories are directly comparable. } \\
\text { Representative of } \\
\text { human malaria }\end{array}$ \\
$\begin{array}{l}\text { probable action of the compound(s) when } \\
\text { used to treat human malaria. }\end{array}$ \\
\hline
\end{tabular}

to prioritize additional screening methods for P. falciparum. However, there is the requirement to continually upgrade the existing assays. With the emergence of artemisinin resistance [18], compounds that, like artemisinins, are active against the early ring stages of the erythrocytic cycle or those that are active against artemisinin-resistant strains of $P$. falciparum should be a priority. A low throughput viability-based assay approach that allows discrimination between compounds based on their anti-malarial mode-ofaction has been developed [19]. Further work is needed to develop more novel screens or further modifications of existing methods.

In vivo models using rodent Plasmodium species or $P$. falciparum in severe combined immunodeficiency (SCID) mice or non-human primates (NHP) are available for pharmacological studies. Mouse models may give species dependent screening results and so different Plasmodium species need to be used in the initial rodent model screening of compounds. Primate models are difficult, of limited availability, and not cost-effective.

There is a need to identify a good in vivo model for severe malaria that can be used in secondary screening. To find appropriate models, the target profile needs to be better defined.

\section{Plasmodium vivax}

Discovery of new drugs active against $P$. vivax blood stage infections is being impaired because of a lack of HTS assays (Table 2). The development of such drugs will be key to achieving malaria control and elimination.

The key challenge is the lack of an in vitro culture system for growing P. vivax [20]. The availability of fresh isolates relies on the collection of infected human blood, which needs to be processed within six hours from collection. Consequently, the laboratory facilities must be beside the patients and in easily accessible locations. In addition, $P$ vivax preferentially invades reticulocytes which are short-lived and represent only approximately 
Table 2 Existing range of in vitro screening methods and in vivo models for blood stage anti-malarials

\begin{tabular}{|c|c|c|c|}
\hline & In vitro pharmacology (HTS Screens) & In vivo pharmacology & Human pharmacology \\
\hline \multirow[t]{9}{*}{ Plasmodium falciparum } & \multirow[t]{2}{*}{ - Radioactive (3H-Hypoxanthine) } & Human parasite models: & \multirow[t]{13}{*}{ Human challenge } \\
\hline & & - P. falciparum in SCID mouse & \\
\hline & \multirow[t]{2}{*}{ - Colorimetric (pLDH, HRPII) } & - Aotus/Saimiri NHP & \\
\hline & & Mouse models: & \\
\hline & - Fluorescent (Sybr Green) & \multirow{2}{*}{$\begin{array}{l}\text { - Plasmodium berghei (including GFP or LuC } \\
\text { reporter strains) }\end{array}$} & \\
\hline & \multirow{4}{*}{$\begin{array}{l}\text { - Transgenic parasite (GFP, Luc reporter } \\
\text { strains) }\end{array}$} & & \\
\hline & & - Plasmodium vinckei & \\
\hline & & - Plasmodium chabaudi & \\
\hline & & - Plasmodium yoelii & \\
\hline \multirow[t]{4}{*}{ Plasmodium vivax } & Invasion assay & Human parasite models & \\
\hline & \multirow{3}{*}{$\begin{array}{l}\text { Flow cytometry (both at present only } \\
\text { medium throughput) }\end{array}$} & - Aotus/ Saimiri NHP & \\
\hline & & Primate models & \\
\hline & & - Plasmodium cynomolgi & \\
\hline
\end{tabular}

$1 \%$ of the circulating red blood cells. New improved ex vivo methods for isolation and cryopreservation of $P$. vivax isolates and of concentrated cord blood reticulocytes have been developed [21] and a short term (24 hour) invasion assay has been standardised and used for both drug sensitivity tests and proteomics [22]. However, the invasion assay still relies on microscopic determination of schizont maturation and reticulocytes invasion and thus is quite laborious and requires trained personnel. More recently, the same group [23] developed a simple, field optimised protocol using fluorescent dyes and flow cytometry to determine the antimalarial drug sensitivity in freshly isolated $P$. vivax: this became possible mostly because portable, low cost cytometers became available. Flow cytometry may become a viable and practical alternative to microscopy, although the problem of long term cultures of $P$. vivax persists and represents a key block to future progress in a number of areas of $P$. vivax research. The development of a SCID mouse model for $P$. vivax could be seen as an alternative.

Virtually all $P$. falciparum blood stage drugs are also active against $P$. vivax, but the levels of sensitivity of each species to the drugs may differ. The workshop concluded that developing primary screens that would pick up vivax activity separately was not a priority. It should be to develop good secondary in vivo models, particularly for compounds that have a differential in vitro activity against both species.

\section{Screening methods against liver stage parasites}

Until recently, validated screening assays for liver stage $P$. falciparum and $P$. vivax liver stages were virtually non-existent, and much work needs to be done to develop appropriate validated methods (Table 3 ).
A major priority for the liver stages of both $P$. falciparum and P. vivax is to develop a reproducible and robust hepatocyte culture system that is highly susceptible to large scale invasion by malaria sporozoites. This should ensure the complete development of the parasite and ideally give good machinery for drug metabolism. Another block is the lack of a robust supply of sporozoites and cryogenic methods to store and transport them.

\section{Plasmodium falciparum}

Rudimentary assays already exist for liver stages that do not form hypnozoites: P. falciparum, Plasmodium yoelii, and Plasmodium berghei [24]. Until recently, only primary hepatocytes appeared suitable for $P$. falciparum and they have very low permissivity for infection [25]. Stable and infectable hepatocyte lines and standardized conditions for culturing hepatocytes need to be developed, because primary human liver cells are of variable quality and lose their differentiation in culture. There is also a priority to find ways of increasing the permissivity of hepatocytes to infection by sporozoites. The recent

Table 3 Existing in vitro and in vivo models against liver stage parasites

\begin{tabular}{llll}
\hline & $\begin{array}{l}\text { In vitro } \\
\text { pharmacology } \\
\text { (HTS Screens) }\end{array}$ & $\begin{array}{l}\text { In vivo } \\
\text { pharmacology }\end{array}$ & $\begin{array}{l}\text { Human } \\
\text { pharmacology }\end{array}$ \\
\hline $\begin{array}{l}\text { Plasmodium } \\
\text { that do }\end{array}$ & P. falciparum & Mouse models & Human \\
not form & P. . berghei & - P. berghei & \\
hypnozoites & - P. yoelii & - P. yoelii & \\
Hypnozoites & - P. vivax in & Primate & \\
-forming & hepatocyte cell lines & models & \\
Plasmodium & - P. cynomolgi in & - P. cynomolgi & \\
species & hepatocytes & & \\
& from Rhesus monkeys & & \\
\end{tabular}


study by Bathia and colleagues that use cryopreserved hepatocytes (described below for $P$. vivax but also applied to $P$. falciparum) is of potential value for all liver stage models [26].

\section{Plasmodium vivax}

The situation with screening for active liver stage activity against $P$. vivax infections is the same as for $P$. falciparum, with the additional challenges of (a) developing methods to cultivate the sexual blood stage (and thus to obtain infective sporozoites following mosquito feed) and (b) developing appropriates tools to distinguish the hypnozoite from the replicating schizont stage. Clearing hypnozoites is critical for malaria elimination.

Cellular assays for the three key steps of liver-stage biology are thus needed: hepatocyte infection, hypnozoite formation, and reactivation to hepatic schizonts. Hypnozoite identification is difficult because of their relatively small size, absence of validated biomarkers and the low infection rate $[4,24]$. A small-scale liver culture system for $P$. vivax, relying on cryopreserved sporozoites has identified primaquine-sensitive small forms [27]. Recently, schizonts and fluorescent uninucleate persisting forms (displaying all the characteristics of hypnozoite-forms) could be characterized for Plasmodium cynomolgi, a simian parasite related to $P$. vivax. These were observed after sporozoite infection of Macaca fascicularis primary hepatocytes $[25,28]$. This simian system provides a more reliable supply of both parasites and primary cells as well as transgenic fluorescent parasites. Another recent study has reported an in vitro model that recapitulates the entire liver stage for both P. falciparum and P. vivax in a microscale human liver platform composed of cryopreserved, micropatterned human primary hepatocytes surrounded by supportive stromal cells. This system allow the release of infected merozoites and infection of overlaid erythrocytes, as well as the establishment of small forms in late liver stages of $P$. vivax [26].

Biomarkers that distinguish hypnozoites from the hepatic schizonts are needed to speed up work on the nature of dormancy. There is very little understanding of the biology of both the liver schizont and the hypnozoite stage of infection. The pressing question is "what is a hypnozoite, how does it function, and how can its quiescent metabolism be targeted?"

The availability of robust and reliable culture models and methods for hypnozoite identification will provide the foundations to obtain key information on the biology of hypnozoite and their reactivation process, paving the way for a quantitative high-throughput liver stage screen [29]. Once appropriate screens have been developed, compounds will be tested first for activity against all hepatic stage in a primary screen and then secondarily screened for hypnozoite activity.

\section{Screening methods against sexual stages}

Transmission blocking (through the sexual and mosquitobased stages of the Plasmodium life cycle) will become more important as malaria control moves towards malaria elimination. It is argued that the number of parasites that need to be killed to break the transmission cycle is orders of magnitude less than is the case in treating infections.

Currently there is no generally accepted detailed target profile for a new chemical entity that would act principally against the sexual stages. Such a profile needs to be developed so that it is clear exactly which stage(s) should be targeted (gametocytes, intermediate stages - gametes, zygotes, ookinetes, oocysts, or finally sporozoites). Recently, a comprehensive review on the next generation of antimalarial drugs needed to control and eliminate malaria has been published and the target candidate profiles (TCP) for transmission blocking agents have been outlined [30].

Most probably conventional blood schizonticides will block the transition from asexual to sexual stages, so screening for drugs that block this transition is not the first priority. However, mature gametocytes are longlived and even patients on treatment may continue to transmit malaria for weeks after clearance of asexual parasites. There is data showing that some anti-malarial drugs can in fact induce gametocytogenesis [31]. Drugs that will kill long-lived stage $\mathrm{V}$ gametocytes and/or extra-erythrocytic parasite stages are needed to sustain the elimination phase. Over the last few years, efforts have been made to develop a reproducible and robust method for producing gametocytes in vitro, and sustain their in vitro differentiation until the long-lasting late stage V. In addition, new assays for gametocytocidal activity have been developed and are presently used for large compounds screening (Table 4) [32]. These assays fall into three major categories:

1. Measurements of parasite DNA content using fluorescent dyes (like hydroethidine) for flow cytometry [34]. The sensitivity is good but the throughput is quite low. High content screening assays for stage IV-V gametocytes are under development [42].

2. Measurement of the metabolic/redox activity of $P$. falciparum gametocytes, using the ATP bioluminescence assay for late stage gametocytes [36,37], the fluorimetric Alamar blue test [39,40], or the colorimetric assay with pLDH [41]. The advantage of all these assays is that they can be used with common laboratory strains or even field isolates since they do not require transgenesis. 
Table 4 Existing in vitro assays for screening compounds against Plasmodium falciparum gametocytes (stage I-V)

\begin{tabular}{ll}
\hline $\begin{array}{l}\text { In vitro pharmacology } \\
\text { L/MTS/HTS Screens (using } \\
\text { non-transgenic parasites) }\end{array}$ & $\begin{array}{l}\text { In vitro pharmacology } \\
\text { MTS/HTS screens (using } \\
\text { transgenic parasites) }\end{array}$ \\
\hline $\begin{array}{l}\text { Microscopic counts of Giemsa } \\
\text { smear (gold standard) }\end{array}$ & Pfs16-GFP transgenic parasites [33]. \\
$\begin{array}{l}\text { Flow cytometry of hydroethidine } \\
\text { labelled gametocytes [34] }\end{array}$ & $\begin{array}{l}\text { Stage-specific luciferase expression } \\
\text { in transgenic parasites [35] }\end{array}$ \\
$\begin{array}{l}\text { ATP- bioluminescence assay for } \\
\text { stage IV-V gametocytes [36,37] }\end{array}$ & $\begin{array}{l}\text { Three transgenic GFP lines, used } \\
\text { for flow cytometry assay [38] }\end{array}$ \\
$\begin{array}{l}\text { Alamar blue, fluorescent redox } \\
\text { indicator [39,40] }\end{array}$ & \\
Parasite lactate dehydrogenase & \\
activity (pLDH) [41] & \\
\hline
\end{tabular}

3. Transgenesis has been applied with success to produce P. falciparum lines expressing GFP or GFPLuc reporter genes active on different stages of gametocyte maturation. They have been used to identify compounds active on early (I-II), middle (III) or late (IV-V) stage gametocytes [35]. The GFP transgenic lines have been used with flow cytometry $[37,38]$. The assays using GFP or GFP-Luc reporter genes appear to be quite sensitive, robust, with a good signal to noise ratio.

\section{Assays against the sporogonic stages in the mosquito}

To achieve the objective of local elimination/eradication of malaria, it is well recognized that malaria transmission through the mosquito vector must be reduced. The standard membrane-feeding assay (SMFA) is the gold standard assay for evaluation of any transmissionblocking intervention, but it has a very low throughput and is expensive.

A series of novel assays (Table 5) that can identify activity across almost all sexual stages have been recently described or are under development. Some of them are still in a medium or low throughput format, but improvements are expected shortly. The P. berghei ookinete development assay is the only one adapted to HTS format [43]. The test is quite robust, and reproducible and has been used in HTS screens of a large compound library (the Tres Cantos Antimalarial Set (TCAMS)) [44].

To verify if stage $\mathrm{V}$ gametocytes are able to continue their differentiation toward male and female gametes, two different assays are available. The $P$. falciparum exflagellation assay is limited to male gametocytes, and although it has recently been semi-automatized by image acquisition of motile 'exflagellation' events, still it is limited by the short time-window of this phenomenon [43].

The production of female gametes can be visualized by morphology on Giemsa-stained smears. The stable expression of unique surface antigens by female gametes
Table 5 Existing assays for screening transmission blocking compounds on the sporogonic stages in mosquitoes

\begin{tabular}{ll}
\hline $\begin{array}{l}\text { Pharmacology } \\
\text { L/MTS/HTS Screens }\end{array}$ & $\begin{array}{l}\text { Plasmodium } \\
\text { species }\end{array}$ \\
\hline $\begin{array}{l}\text { Male exflagellation assay [45] } \\
\text { Female activation assay (Delves, } \\
\text { unpublished) }\end{array}$ & $\begin{array}{l}\text { P. falciparum } \\
\text { P. falciparum }\end{array}$ \\
$\begin{array}{l}\text { Standard membrane feeding assay } \\
\text { (SMFA) [46,47] }\end{array}$ & P. falciparum \\
SMFA using PbGFP CON [48] & P. berghei \\
$\begin{array}{l}\text { P. berghei ookinete development } \\
\text { assay [43] }\end{array}$ & Anopheles stephensi \\
GFP-luc sporozoites in mosquito salivary & P. berghei (transgenic) \\
glands [49] & P. berghei (transgenic) \\
\hline
\end{tabular}

has offered the possibility to develop MTS/HTS assays of macrogametogenesis (Delves et al. unpublished).

Finally, the availability of GFP-tagged lines is transforming the throughput of the SMFA assay [46], one of the most sensitive, albeit very low throughput, assay to evaluate transmission-blocking compounds [48]. Counting GFP-Luc expressing salivary gland sporozoites in mosquito lysate is also possible, although with limited sensitivity at present and only in the P. berghei model [49].

Drugs targeted solely at the late sexual stages will need to reside in the human body for at least five to nine days after the appearance of symptoms. This is in order to be present when the mature gametocytes (Stage V) start to appear in the blood. This has significant implications for the bioavailability and pharmacokinetic properties of drug candidates (see "Priorities for the development of enabling technologies”).

\section{Priorities for the development of enabling technologies Blood stages}

- Understanding the technical problems behind the development of a P. vivax blood stage culture system and overcoming them.

- Development of a P. vivax blood stage rodent model.

\section{Liver stages}

- Development of a robust hepatocyte cell system to enable study of $P$. falciparum \& $P$. vivax liver stages.

- Development of a standardised and improved culture system for the study of $P$. falciparum $\& P$. vivax liver stages.

- Understanding of the nature and biology of hypnozoites with the objective of identifying a 
biomarker for drug activity against this stage of $P$. vivax infection.

- Development of a robust and reliable supply of falciparum and vivax sporozoites and a method for safely transporting them between laboratories.

- Investigate the possibility of using a liver schizont assay system as a surrogate for activity against hypnozoites in primary screening for novel antivivax drugs.

\section{Sexual stages}

- Development of a target product profile for a drug to target the sexual stages of P. falciparum.

- Development of robust screening methods for stage IV-V gametocytes of $P$. falciparum

- Development of a P. falciparum (and P. vivax) ookinete assay to complement the current $P$ berghei assay.

- Development of male and female gamete activation assays

- Development of MTS/HTS for SMFA assay

\section{Identifying novel targets}

There is a clear need for the identification and development of new classes of anti-malarials, i.e. belonging to novel chemical series and with novel modes of action. The current reality is that much drug discovery is still focused on a limited number of historic targets (primarily the folate and haemoglobin degradation pathways), and primarily on the blood stage of the parasite's life cycle. There is a wealth of untapped targets in the various developmental stage proteomes that might be targeted.

Databases on genes and metabolic pathways offer opportunities to identify possible new target-based approaches. The cross-correlation of this information can be a very valuable filter (e g, identifying genes that are active in both gametocytes and in the blood stages). Orthology-based screening between Plasmodium species may also give valuable insights into promising new targets. The high level of conservation between P. falciparum and $P$. vivax genomes indicates that novel targets for vivax might be established from proven falciparum targets.

As the effort against malaria moves from the control phase to elimination, parts of the parasite life cycle beyond the blood stages will need to be targeted. Liver stages, gametocytes and sporozoites were highlighted in the Workshop as being potentially important for the elimination phase.

\section{Targeting erythrocytic schizogony}

In the control phase, the focus is on the treatment of symptomatic patients, i.e., treating asexual blood stages.

\section{Finding novel drug classes}

One approach is the mining of genomic databases to identify novel targets (see below), followed by the screening of high diversity chemical libraries on such targets. Another strategy consists of first using such libraries (including libraries of natural products) in phenotypic screens to constitute the so-called "malaria boxes", and subsequently identify in secondary screens the molecular targets of compounds with parasiticidal activity. Several million chemical entities have been screened for anti-malarial activity, based on whole cell screens of P. falciparum. The screens have included fully synthetic (from both commercially available and pharmaceutical industry proprietary sources) and natural product libraries. More than 20,000 compounds are now available that are known to have selective parasiticidal activity, but whose molecular targets are unknown [50]. Many of these are likely to act through novel pharmacophores on novel molecular targets. A wider range of libraries should be investigated, including compound banks from agrochemical companies. The accompanying paper outlines the CRIMALDDI Consortium's recommendations to expedite the use of this valuable source of information [7].

\section{Finding novel targets}

The characteristics of a good target should include:-

"Must have" characteristics:

- Compound should be able to kill the parasite (be "cidal") in much less than 48 hours through either inhibition of the target or interaction of the compound with the target;

- The target should be validated either chemically or genetically, both in vitro and in vivo;

- The target should be druggable, i.e. should be amenable to treatment with small molecules at low concentrations;

- There needs to be a reliable functional assay of the target.

"Nice to have" characteristics:

- Lack of orthology of the target in humans;

- Cellular assay of the effect of compounds on the target;

- A crystal structure of the target.

\section{Considerations about the various stages in erythrocytic schizogony}

A key requirement in most target product profiles is rapid parasite kill. The speed-of-action of the artemisinins is attributed to their activity throughout the erythrocytic stage (in contrast, most other anti-malarials that are active 
only in the trophozoite stage). Similarly, interfering with the schizont stage and interrupting the release of merozoites are potentially promising but unexploited strategies.

Other processes identified for further biological understanding that might lead to novel targets of most value for drug discovery were:-

- Ring stage particularly focussing on Merozoite invasion: formation of the parasitophorous vacuole and associated transport pathways.

- Trophozoite stage: Metabolic pathway characterisation in the trophozoite (including haemoglobin degradation) and host cell remodelling.

- Schizonts: Nuclear division, merozoite ontogeny, egress mechanisms.

In the absence of robust culture systems it is reasonable at this stage to assume $P$. falciparum will be a suitable model for blood stage $P$. vivax infections in many cases. However, additional work is needed to fully validate this assumption on a hit by hit basis.

\section{Host cell targets}

Recent research has identified an essential role for host erythrocyte enzymes in parasite development $[51,52]$. A number of the parasiticidal compounds may target a host cell enzyme rather than a molecule encoded by the parasite. Targeting host cell (erythrocyte or hepatocyte) enzymes that are required for parasite survival is an additional, as yet unexploited, therapeutic strategy. Recent research indicates that such potential host cell targets include enzymes that are well-established targets in other pathologies (e g, protein kinases in cancer). A "piggy-back" approach might be implemented that would considerably speed up discovery/development. Additional fundamental research must be performed to validate this approach.

\section{Targeting liver stages}

The highest priority in this area is to understand the biology of liver stage parasites, especially the hypnozoites that act as a reservoir for $P$. vivax. Drug activity against hypnozoites will become crucial during elimination. At present even the exact definition of a hypnozoite is not clear. Without such understanding, practical screening techniques cannot be developed and modes of action will be poorly understood. A high priority is to develop simple markers to identify hypnozoites from active, infected hepatocytes (preferably in human liver cells). This would be a key step to developing screens against the dormant stages.
Priority should also be given to the following aspects of the infected hepatocytes:

- Reactivation of hypnozoites in an in vitro model to allow testing of compounds which block reactivation or kill the dormant cells;

- Causes of dormancy and the reasons for liver stage parasites to "choose" between the active and dormant state.

Some insights or leads that may be starting points for research were identified:-

- Known efficacy of 8-aminoquinolines (primaquine and tafenoquine) against hypnozoites;

- The use of bio-informatics to identify potentially important genes and metabolic pathways;

- Non-haem targets in the blood stage;

- Effect of antibiotic pre-treatment of apicoplasts.

\section{Targeting sexual development and sporogony}

Gametocytes are an interesting point to interrupt transmission of the parasite. This could be either the sole activity or an additional activity from a compound that is acting elsewhere in the life cycle. There will be some ethical and practical issues: a purely anti-gametocyte drug would not directly benefit the patient and so would impose a high safety requirement on the compound. On the other hand, a drug targeting gametocytes exclusively (but not affecting asexual stages) would be a valuable asset in preventing the escape of genotypes that confer resistance against anti-asexual drugs: thus one of the main benefits of transmission-blocking drugs would be to protect co-administered curative drugs against resistance development and spread. The mechanisms of action of existing, blood stage, active compounds against gametocytes should be investigated.

Recent research has identified crucial roles for several enzymes in sexual development. Possible target processes to attack gametocytes include:

- Gametocytogenesis: a process with many metabolic processes active and so offering multiple points to disrupt the parasite;

- Egress and subsequent gametogenesis mechanisms (especially proteases, protein kinases, and lipid metabolism);

- The mode of action of primaquine against gametocytes and whether it has a common biochemical target at all stages of the parasite life cycle.

Similarly, drugs that have activity against sporozoites might be of value. Sporozoite-active compounds would 
act in many ways like a parasite repellent. The target product profile should clarify how this type of activity will be of demonstrable value in the field. It will be useful to screen against sporozoites all the current screening hits to see if there is this additional activity to that against blood and/or liver stages.

\section{Natural products}

The chemical diversity derived from natural products is tremendous. Natural products are an "exquisite" source of new drugs as Nature has screened orders of magnitude of more compounds than has been possible with HTS. Many current anti-malarials originated as natural products. However in recent years no compounds in development have originated directly from a natural product. Many natural products have shown potent antiplasmodial effects but have not been progressed [53]. Recent developments (such as the availability of the whole parasite genome) warrant a re-visit of the natural product route of drug discovery vis-à-vis target identification. Natural products generally span a different chemical space than synthetic compounds. In addition, natural products can be bio-available with properties outside conventional Lipinski space and they have evolved to interact with biological molecules. Therefore, screening of natural products in whole cell assays are of potentially great value in identifying new druggable targets that would be rejected if the Lipinski rules were blindly followed $[54,55]$. They will also be of value in identifying polypharmacological modes of action. The metabolic activity identified with natural products can also be used to define empirical targets without initially establishing a protein target [56]. The protein/target may be determined later in the development process. Naturally occurring "libraries" of natural products should not be ignored, both in seeking novel chemical classes and as tools to identify novel targets. They may have a better chance to produce hits in drug screening because they have evolved to interact with biological targets. Another approach by which natural products may be useful in the identification of druggable targets is activity-based protein profiling [57]. Proteins/enzymes identified in this way can then be investigated further as potential therapeutic targets by both classical mechanism of action studies or reverse pharmacology [58].

The shared ancestry of apicoplexans and algae suggest that new "plant-like" targets exist beyond the apicoplast. This may be of value in identifying leads for less metabolically active stages in the parasite life cycle (e g, gametocytes, hypnozoites). The problem of the regulatory pathway with natural product mixtures was noted. The drive for low-cost drugs also was a problem for natural products, which are often complex molecules and have potentially high costs of manufacture. However, better semi-synthetic approaches (as has is being used for artemisinin) may reduce this problem.

\section{Conclusion}

The ambitious target of malaria elimination will only be achieved with the development of new drugs carefully targeted at the key challenges that exist for both control and elimination. The CRIMALDDI Project aimed to identify the most important approaches that should be adopted, as a guide to the various organizations - policy makers, funders, and researchers - on where to prioritize their efforts.

It is clear from the recommendations of the two Workshops described in this paper that a very pressing need is to develop the new and improved enabling technologies that allow new drugs with the right target profiles to be identified quickly. These novel technologies are often forgotten in the understandable drive towards the development of new medicines. At a time of limited resources, it is important that drug discovery efforts are clearly focused on the areas of greatest need and/or which show the greatest probability of success. Better enabling technologies will assist in this prioritization, as well as helping to identify novel approaches and enabling them to be studied more effectively.

Exploring a more diverse range of potential drug targets is a priority. These may be better able to address the shortcomings of the current range of anti-malarials. A broader approach that explores of the entire parasite's life cycle is recommended, as this holds the promise both of addressing current challenges and meeting the requirements of drugs for elimination [29].

The work of the CRIMALDDI Project described here and in related papers [5,7] will hopefully provide some guidance to policy-makers, funders, and researchers in prioritizing their malarial drug discovery efforts. It can also draw attention to the importance of the interplay between the existence of new technologies and the malaria community's ability to move beyond the current portfolio of well-characterized and studied targets. It should add to the global discussion on how best to prioritize scarce resources, especially as the community moves into the postMillennium Development Goal period and an updated Global Malaria Action Plan is being developed.

\footnotetext{
Abbreviations

CRIMALDDI: The coordination, rationalization, and integration of antimalarial drug discovery \& development initiatives; EU: European Union; HTS: Highthroughput screening; MMV: Medicines for malaria venture; MTS: Mediumthroughput screening; NHP: Non-human primate; SCID: Severe combined immunodeficiency; SMFA: Standard membrane feeding assay; TDR: UNICEF/ UNDP/World Bank/WHO Special Programme for Tropical Disease Research \& Training; TPP: Target product profile.
}

\section{Competing interests}

The authors declare that they have no competing interests. 


\section{Authors' contributions}

$H V, D T, C D$, and KC prepared the first draft of paper. ICB subsequently edited it. All authors contributed to the preparation of the paper. All authors have read and approved the final manuscript.

\section{Acknowledgements}

The Consortium would like to acknowledge the support of the European Union FP7 grant that has made the project possible. The Consortium would like to acknowledge the valuable input of the participants in the two workshops that developed the recommendations in this paper: Geneva, Switzerland (May 2010): Stage-specific screening methods lan Boulton (TropMed Pharma Consulting, UK), Foluke Fakorede (TDR, Switzerland), Jose Garcia-Bustos (GlaxoSmithKline, Spain), Annette Habluetzel (Università di Camerino, Italy), Marcel Kaiser (Swiss Tropical and Public Health Institute, Switzerland), Sumalee Kamchonwongpaisan (National Centre for Genetic Engineering \& Biotechnology, Thailand), Clemens Kocken (Biomedical Primate Research Centre, Netherlands), Didier Leroy (Medicines for Malaria Venture, Switzerland), Louis Maes (University of Antwerp, Belgium), Maria Manuel Mota (Universidade de Lisboa, Portugal), Maurizio Muraca (Ospedale Pediatrico Bambino Gesù, Italy), Solomon Nwaka (TDR, Switzerland), Bernadette Ramirez (TDR, Switzerland), Tracy Seddon (Liverpool School of Tropical Medicine, UK), Robert Sinden (Imperial College, UK), Georges Snounou (University Pierre et Marie Curie/INSERM, France), Roberta Spaccapelo (Università di Perugia, Italy), Donatella Taramelli (Università di Milano, Italy), Chairat Uthaipibull (National Centre for Genetic Engineering \& Biotechnology, Thailand), Henri Vial (Centre National de la Recherché Scientifique, University of Montpellier, France), Sergio Wittlin (Swiss Tropical and Public Health Institute, Switzerland).

Liverpool, UK (October 2010): Plasmodium falciparum and Plasmodium vivax Novel Targets and Classes

Pietro Alano (Istituto Superiore di Sanità, Italy), Giancarlo Biagini (Liverpool School of Tropical Medicine, UK), lan Boulton (TropMed Pharma Consulting, UK), Kelly Chibale (University of Cape Town, South Africa), Christian Doerig (EPFL-INSERM, Switzerland), Hagai Ginsburg (Hebrew University of Jerusalem, Israel), Dean Goodman (University of Melbourne, Australia), Susan Jones (Liverpool School of Tropical Medicine, UK), Clemens Kocken (Biomedical Primate Research Centre, Netherlands), Didier Leroy (Medicines for Malaria Venture, Geneva), Alexis Nzila (University of Cape Town, South Africa), David Roos (University of Pennsylvania, USA), Phil Rosenthal (University of California, San Francisco, USA), llaria Russo (University of Perugia, Italy), Jonathon Vennestrom (University of Nebraska, USA), Steve Ward (Liverpool School of Tropical Medicine, UK), Andrew Wilks (Monash University, Australia), Paul Wyatt (University of Dundee, UK)

\section{Author details}

${ }^{1}$ Centre National de la Recherche Scientifique, UMR 5235, Université Montpellier 2, Place Eugene Bataillon, 34095 Montpellier, Cedex 5, France. ${ }^{2}$ Dipartmento di Scienze Farmacologiche e Biomolecolari, Università di Milano, Via Pascal 36, 20133 Milano, Italy. ${ }^{3}$ TropMed Pharma Consulting Ltd, 10 Brampton Chase, Lower Shiplake, Oxfordshire RG9 3BX, UK. ${ }^{4}$ Liverpool School of Tropical Medicine, Pembroke Place, Liverpool L3 5QA, UK. ${ }^{5}$ Department of Microbiology, School of Biomedical Sciences, Monash University, Wellington Road, Clayton, VIC 3800, Australia. ${ }^{6}$ Department of Chemistry, University of Cape Town, Rondebosch, Cape Town 7701, South Africa.

Received: 17 July 2013 Accepted: 22 October 2013

Published: 05 November 2013

\section{References}

1. Alonso PL, Brown G, Arevalo-Herrera M, Binka F, Chitnis C, Collins F, Doumbo OK, Greenwood B, Hall BF, Levine MM, Mendis K, Newman RD, Plowe CV, Rodríguez MH, Sinden R, Slutsker L, Tanner M: A research agenda to underpin malaria eradication. PLoS Med 2011, 8:e1000406.

2. Roll Back Malaria Partnership: Global malaria action plan. Geneva: RBM Partnership: 2008.

3. Sinden RE: A biologist's perspective on malaria vaccine development. Hum Vaccin 2010, 6:3-11

4. Wells TN, Burrows JN, Baird JK: Targeting the hypnozoite reservoir of Plasmodium vivax: the hidden obstacle to malaria elimination. Trends Parasitol 2010, 26:145-151.
5. Boulton IC, Nwaka S, Bathurst I, Lanzer M, Taramelli D, Vial H, Doerig C, Chibale K, Ward SA: CRIMALDDI: a co-ordinated, rational, and integrated effort to set logical priorities in anti-malarial drug discovery initiatives. Malar J 2010, 9:202.

6. CRIMALDDI: The Coordination, Rationalization, and Integration of Antimalarial Drug Discovery \& Development Initiatives. http://www.crimalddi.eu.

7. Ward SA, Boulton IC: CRIMALDDI: a prioritized research agenda to expedite the discovery of new anti-malarial drugs. Malar J 2013, 12:395.

8. Ridley RG: Medical need, scientific opportunity and the drive for antimalarial drugs. Nature 2002, 415:686-693.

9. Woodrow CJ, Dahlström S, Cooksey R, Flegg JA, Le Nagard H, Mentré F, Murillo C, Ménard D, Nosten F, Sriprawat K, Musset L, Quashie NB, Lim P. Fairhurst RM, Nsobya SL, Sinou V, Noedl H, Pradines B, Johnson JD, Guerin PJ, Sibley CH, Le Bras J: High-throughput analysis of antimalarial susceptibility data by the worldwide antimalarial resistance network (WWARN) in vitro analysis and reporting tool. Antimicrob Agents Chemother 2013, 57:3121-3130.

10. Desjardins RE, Canfield CJ, Haynes JD, Chulay JD: Quantitative assessment of antimalarial activity in vitro by a semiautomated microdilution technique. Antimicrob Agents Chemother 1979, 16:710-718.

11. Makler MT, Ries JM, Williams JA, Bancroft JE, Piper RC, Gibbins BL, Hinrichs DJ: Parasite lactate dehydrogenase as an assay for Plasmodium falciparum drug sensitivity. Am J Trop Med Hyg 1993, 48:739-741

12. Noedl $H$, Wongsrichanalai $C$, Wernsdorfer WH: Malaria drug-sensitivity testing: new assays, new perspectives. Trends Parasitol 2003, 19:175-181.

13. Wein S, Maynadier M, Tran Van Ba C, Cerdan R, Peyrottes S, Fraisse L, Vial H: Reliability of antimalarial sensitivity tests depends on drug mechanisms of action. J Clin Microbiol 2010, 48:1651-1660.

14. Smilkstein M, Sriwilaijaroen N, Kelly JX, Wilairat P, Riscoe M: Simple and inexpensive fluorescence-based technique for high-throughput antimalarial drug screening. Antimicrob Agents Chemother 2004, 48:1803-1806.

15. Cui L, Miao J, Wang J, Li Q, Cui L: Plasmodium falciparum: development of a transgenic line for screening antimalarials using firefly luciferase as the reporter. Exp Parasitol 2008, 120:80-87.

16. Helm S, Lehmann C, Nagel A, Stanway RR, Horstmann S, Llinas M, Heussler $V T$ : Identification and characterization of a liver stage-specific promoter region of the malaria parasite Plasmodium. PLoS One 2010, 5:e13653.

17. Lucumi E, Darling C, Jo H, Napper AD, Chandramohanadas R, Fisher N, Shone AE, Jing H, Ward SA, Biagini GA, DeGrado WF, Diamond SL, Greenbaum DC: Discovery of potent small-molecule inhibitors of multidrug-resistant Plasmodium falciparum using a novel miniaturized high-throughput luciferase-based assay. Antimicrob Agents Chemother 2010, 54:3597-3604

18. Dondorp AM, Nosten F, Yi P, Das D, Phyo AP, Tarning J, Lwin KM, Ariey F, Hanpithakpong W, Lee SJ, Ringwall P, Silamut K, Imwong M, Chotivanich K, Lim P, Herdman T, An SS, Yeung S, Singhasivanon P, Day NPJ, Lindegardh $\mathrm{N}$, Socheat D, White NJ: Artemisinin resistance in Plasmodium falciparum malaria. N Engl J Med 2009, 361:455-467.

19. Sanz LM, Crespo B, De-Cózar C, Ding XC, Llergo JL, Burrows JN, GarcíaBustos JF, Gamo F-J: P. falciparum in vitro killing rates allow to discriminate between different antimalarial mode-of-action. PLoS One 2012, 7:e30949.

20. Russell B, Suwanarusk R, Malleret B, Costa FT, Snounou G, Baird JK, Nosten F, Rénia L: Human ex vivo studies on asexual Plasmodium vivax: the best way forward. Int J Parasitol 2012, 42:1063-1070.

21. Borlon C, Russell B, Sriprawat K, Suwanarusk R, Erhart A, Renia L, Nosten F, D'Alessandro U: Cryopreserved Plasmodium vivax and cord blood reticulocytes can be used for invasion and short term culture. Int J Parasitol 2012, 42:155-160.

22. Russell B, Suwanarusk R, Borlon $C$, Costa FT, Chu CS, Rijken MJ, Sriprawat $K$, Warter L, Koh EG, Malleret B, Colin Y, Bertrand O, Adams JH, D'Alessandro U, Snounou G, Nosten F, Rénia L: A reliable ex vivo invasion assay of human reticulocytes by Plasmodium vivax. Blood 2011, 118:e74-81.

23. Russell B, Malleret B, Suwanarusk R, Anthony C, Kanlaya S, Lau Y, Woodrow C, Nosten F, Renia L: Field based flow cytometry for ex vivo characterization of Plasmodium vivax and $P$. falciparum antimalarial sensitivity. Antimicrob Agents Chemother 2013, 57:5170-5174.

24. Gego A, Silvie O, Franetich JF, Farhati K, Hannoun L, Luty AJ, Sauerwein RW, Boucheix C, Rubinstein E, Mazier D: New approach for high-throughput screening of drug activity on Plasmodium liver stages. Antimicrob Agents Chemother 2006, 50:1586-1589. 
25. Dembele L, Gego A, Zeeman AM, Franetich JF, Silvie O, Rametti A, Le Grand R, Dereuddre-Bosquet N, Sauerwein R, van Gemert GJ, Vaillant JC, Thomas AW, Snounou G, Kocken CH, Mazier D: Towards an in vitro model of Plasmodium hypnozoites suitable for drug discovery. PLoS One 2011, 6:e18162.

26. March S, Ng S, Velmurugan S, Galstian A, Shan J, Logan DJ, Carpenter AE, Thomas D, Sim BK, Mota MM, Hoffman SL, Bhatia SN: A Microscale Human Liver Platform that Supports the Hepatic Stages of Plasmodium falciparum and vivax. Cell Host Microbe 2013, 14:104-115.

27. Chattopadhyay R, Velmurugan S, Chakiath C, Andrews Donkor L, Milhous W, Barnwell JW, Collins WE, Hoffman SL: Establishment of an in vitro assay for assessing the effects of drugs on the liver stages of Plasmodium vivax malaria. PLoS One 2010, 5:e14275.

28. Voorberg-van der Wel A, Zeeman AM, van Amsterdam SM, van den Berg A, Klooster EJ, Iwanaga S, Janse CJ, van Gemert GJ, Sauerwein R, Beenhakker N, Koopman G, Thomas AW, Kocken CH: Transgenic fluorescent Plasmodium cynomolgi liver stages enable live imaging and purification of malaria hypnozoite-forms. PLoS One 2013, 8:e54888.

29. Meister S, Plouffe DM, Kuhen KL, Bonamy GM, Wu T, Barnes SW, Bopp SE, Borboa R, Bright AT, Che J, Cohen S, Dharia NV, Gagaring K, Gettayacamin M, Gordon P, Groessl T, Kato N, Lee MC, McNamara CW, Fidock DA, Nagle A, Nam TG, Richmond W, Roland J, Rottmann M, Zhou B, Froissard P, Glynne RJ, Mazier D, Sattabongkot J, Schultz PG, Tuntland T, Walker JR, Zhou Y, Chatterjee A, Diagana T, Winzeler EA: Imaging of Plasmodium liver stages to drive next-generation antimalarial drug discovery. Science 2011, 334:1372-1377.

30. Burrows J, Hooft van Huijsduijnen R, Mohrle J, Oeuvray C, Wells T: Designing the next generation of medicines for malaria control and eradication. Malar J 2013, 12:187.

31. Bousema T, Drakeley C: Epidemiology and infectivity of Plasmodium falciparum and Plasmodium vivax gametocytes in relation to malaria control and elimination. Clin Microbiol Rev 2011, 24:377-410.

32. Lucantoni $L$, Avery $\mathrm{V}$ : Whole-cell in vitro screening for gametocytocidal compounds. Future Med Chem 2012, 4:2337-2360.

33. Peatey CL, Skinner-Adams TS, Dixon MW, McCarthy JS, Gardiner DL, Trenholme KR: Effect of antimalarial drugs on Plasmodium falciparum gametocytes. J Infect Dis 2009, 200:1518-1521.

34. Chevalley S, Coste A, Lopez A, Pipy B, Valentin A: Flow cytometry for the evaluation of anti-plasmodial activity of drugs on Plasmodium falciparum gametocytes. Malar J 2010, 9:49.

35. Adjalley SH, Johnston GL, Li T, Eastman RT, Ekland EH, Eappen AG, Richman A, Sim BK, Lee MC, Hoffman SL, Fidock DA: Quantitative assessment of Plasmodium falciparum sexual development reveals potent transmissionblocking activity by methylene blue. Proc Natl Acad Sci USA 2011, 108:E1214-1223.

36. Lelievre J, Almela MJ, Lozano S, Miguel C, Franco V, Leroy D, Herreros E: Activity of clinically relevant antimalarial drugs on Plasmodium falciparum mature gametocytes in an ATP bioluminescence "transmission blocking" assay. PLoS One 2012, 7:e35019.

37. Peatey CL, Spicer TP, Hodder PS, Trenholme KR, Gardiner DL: A highthroughput assay for the identification of drugs against late-stage Plasmodium falciparum gametocytes. Mol Biochem Parasitol 2011 180:127-131.

38. Buchholz K, Burke TA, Williamson KC, Wiegand RC, Wirth DF, Marti M: A high-throughput screen targeting malaria transmission stages opens new avenues for drug development. J Infect Dis 2011, 203:1445-1453.

39. Tanaka TQ, Williamson KC: A malaria gametocytocidal assay using oxidoreduction indicator, alamarBlue. Mol Biochem Parasitol 2011, 177:160-163.

40. Tanaka TQ, Dehdashti SJ, Nguyen D-T, McKew JC, Zheng W, Williamson KC A quantitative high throughput assay for identifying gametocytocidal compounds. Mol Biochem Parasitol 2013, 188:20-25.

41. D'Alessandro S, Silvestrini F, Dechering K, Corbett Y, Parapini S, Timmerman M, Galastri L, Basilico N, Sauerwein R, Alano P, Taramelli D: A Plasmodium falciparum screening assay for anti-gametocyte drugs based on parasite lactate dehydrogenase detection. J Antimicrob Chemother 2013, 68:2048-2058.

42. Duffy S, Avery VM: Development and optimization of a novel 384-well anti-malarial imaging assay validated for high-throughput screening. Am J Trop Med Hyg 2012, 86:84-92.
43. Delves MJ, Ramakrishnan C, Blagborough AM, Leroy D, Wells TNC, Sinden RE: A high-throughput assay for the identification of malarial transmissionblocking drugs and vaccines. Int J Parasitol 2012, 42:999-1006.

44. Sinden RE, Blagborough AM, Churcher T, Ramakrishnan C, Biswas S, Delves MJ: The design and interpretation of laboratory assays measuring mosquito transmission of Plasmodium. Trends Parasitol 2012, 28:457-465.

45. Delves M, Plouffe D, Scheurer C, Meister S, Wittlin S, Winzeler EA, Sinden RE, Leroy D: The activities of current antimalarial drugs on the life cycle stages of Plasmodium: a comparative study with human and rodent parasites. PLoS Med 2012, 9:e1001169.

46. van der Kolk M, De Vlas SJ, Saul A, van de Vegte-Bolmer M, Eling WM, Sauerwein RW: Evaluation of the standard membrane feeding assay (SMFA) for the determination of malaria transmission-reducing activity using empirical data. Parasitology 2005, 130:13-22.

47. Churcher TS, Blagborough AM, Delves M, Ramakrishnan C, Kapulu MC, Williams AR, Biswas S, Da DF, Cohuet A, Sinden RE: Measuring the blockade of malaria transmission-an analysis of the standard membrane feeding assay. Int J Parasitol 2012, 42:1037-1044.

48. Franke-Fayard B, Trueman H, Ramesar J, Mendoza J, van der Keur M, van der Linden $\mathrm{R}$, Sinden RE, Waters AP, Janse CJ: A Plasmodium berghei reference line that constitutively expresses GFP at a high level throughout the complete life cycle. Mol Biochem Parasitol 2004, 137:23-33.

49. Ramakrishnan C, Rademacher A, Soichot J, Costa G, Waters AP, Janse CJ, Ramesar J, Franke-Fayard BM, Levashina EA: Salivary gland-specific P. berghei reporter lines enable rapid evaluation of tissue-specific sporozoite loads in mosquitoes. PLoS One 2012, 7:e36376.

50. Gamo F, Sanz L, Vidal J, de Cozar C, Alvarez E, Lavandera J, Vanderwall D, Green D, Kumar V, Hasan S, Brown JR, Peishoff CE, Cardon LR, Garcia-Bustos JF: Thousands of chemical starting points for antimalarial lead identification. Nature 2010, 465:305-310

51. Sicard A, Semblat JP, Doerig C, Hamelin R, Moniatte M, Dorin-Semblat D, Spicer JA, Srivastava A, Retzlaff S, Heussler V, Waters AP, Doerig C: Activation of a PAK-MEK signalling pathway in malaria parasite-infected erythrocytes. Cell Microbiol 2011, 13:836-845.

52. Murphy SC, Harrison T, Hamm HE, Lomasney JW, Mohandas N, Haldar K. Erythrocyte $\mathrm{G}$ protein as a novel target for malarial chemotherapy. PLoS Med 2006, 3:e528.

53. Guantai E, Chibale K: How can natural products serve as a viable source of lead compounds for the development of new/novel anti-malarials? Malar J 2011, 10:S2.

54. Cheng KW, Wong CC, Wang M, He QY, Chen F: Identification and characterization of molecular targets of natural products by mass spectrometry. Mass Spectrom Rev 2010, 29:126-155.

55. Pucheault M: Natural products: chemical instruments to apprehend biological symphony. Org Biomol Chem 2008, 6:424-432.

56. Holmes E: The evolution of metabolic profiling in parasitology. Parasitology 2010, 137:1437-1449.

57. Harvey AL: Natural products in drug discovery. Drug Discov Today 2008 13:894-901.

58. Bottcher T, Pitscheider M, Sieber SA: Natural products and their biological targets: proteomic and metabolomic labeling strategies. Angew Chem Int Ed Engl 2010, 49:2680-2698.

doi:10.1186/1475-2875-12-396

Cite this article as: Vial et al.: CRIMALDDI: platform technologies and novel anti-malarial drug targets. Malaria Journal 2013 12:396. 